\title{
Protective effect of trimetazidine on myocardial mitochondrial function in an ex-vivo model of global myocardial ischemia
}

\author{
Pedro Monteiro ${ }^{\mathrm{a}, \mathrm{b}}$, Ana I. Duarte ${ }^{\mathrm{c}}$, Lino M. Gonçalves ${ }^{\mathrm{b}, \mathrm{c}, *}$, \\ António Moreno $^{\mathrm{c}}$, Luís A. Providência ${ }^{\mathrm{b}, \mathrm{c}}$ \\ ${ }^{a}$ Basic Research Unit in Cardiology, Cardiology Department, Coimbra University Hospital, Coimbra, Portugal \\ ${ }^{\mathrm{b}}$ Coimbra University, Medical School, Portugal \\ ${ }^{\mathrm{c}}$ Centre for Neuroscience and Cell Biology of Coimbra, Portugal
}

Received 11 June 2004; received in revised form 26 August 2004; accepted 1 September 2004

Available online 5 October 2004

\begin{abstract}
Trimetazidine is an anti-ischemic drug whose cytoprotective mechanisms are not yet fully understood (but until now mainly related to the trimetazidine-induced "metabolic shift" from lipid $\beta$-oxidation to glucose aerobic oxidation). We studied the effect of trimetazidine on the mitochondrial function of ischemic Wistar rat hearts perfused with glucose, using a model of ex-vivo perfusion (Langendorff system). We measured the electrical potential of the mitochondrial membrane, $\mathrm{O}_{2}$ consumption by the respiratory chain, energy charges generated and the enzyme activities of the respiratory chain complexes. In this model, trimetazidine had a preferential action on the oxidative system (mainly on complex I), increasing its enzyme activity and decreasing $\mathrm{O}_{2}$ consumption after phosphorylation; this could decrease oxygen free radical production and increase mitochondrial integrity, thus allowing the maintenance of the electrical potential. These results allow us to better understand the cytoprotective effects of trimetazidine in coronary artery disease.
\end{abstract}

(C) 2004 Elsevier B.V. All rights reserved.

Keywords: Ischemia; Mitochondrion; Trimetazidine; Perfusion; Glucose

\section{Introduction}

Trimetazidine (1-[2, 3 4-trimethoxybenzyl)-piperazine dihydrochloride), was first used to treat angina in 1964. Trimetazidine is a known metabolic anti-ischemic agent with no significant hemodynamic effects. Clinically, trimetazidine decreases ischemia during coronary angioplasty (Kober et al., 1992) and cardiac surgery (Fabiani et al., 1992) and there is clear evidence that it can improve exercise tolerance in patients with coronary artery disease (Sellier et al., 1987),

\footnotetext{
This work was performed at the Basic Research Unit in Cardiology, in the Centre for Neuroscience and Cell Biology of Coimbra, located in Azinhaga de Santa Comba, 3000 Coimbra, Portugal.

* Corresponding author. Cardiology Department, Coimbra University Hospital, Praceta Prof. Mota Pinto, 3000-Coimbra, Portugal. Tel.: +351 239 400414, +351239 721153; fax: +351239780552.

E-mail address: lgoncalv@ci.uc.pt (L.M. Gonçalves).
}

showing a similar efficacy to propranolol (Detry et al., 1994) or nifedipine (Dalla-Volta et al., 1990). Despite these clinical benefits, the mechanisms involved in its protective effects are not completely understood.

The major source of energy for the cardiac myocyte during aerobic conditions is fatty acid oxidation (Stanley et al., 1997). However, during ischemia the lack of oxygen partially shifts metabolism from fatty acid to glucose oxidation (Veitch et al., 1995). At least part of the protective effects of trimetazidine during ischemia can be explained by enhancement of the "metabolic shift", from lipid oxidation to glucose oxidation (Sentex et al., 1998). This metabolic shift can be explained by an increase in pyruvate dehydrogenase activity (Stanley et al., 1997; Kantor et al., 2000) and by the inhibition of 3-ketoacetyl coenzyme A thiolase (3-KAT) (Kantor et al., 2000).

In hearts not submitted to ischemia, trimetazidine induces a decrease in complex I-mediated $\mathrm{O}_{2}$ consumption during 
state 3 of mitochondrial respiration, which has been interpreted as possible evidence of a specific inhibition of complex I in non-ischemic mitochondria (Stanley et al., 1997). However, this effect was only observed using high concentrations of trimetazidine $(\geq 100 \mu \mathrm{M})$ and was not confirmed by other authors using ex-vivo animal models (Kantor et al., 2000).

Using a model of hypoxia-reoxygenation, Fantini and coworkers have demonstrated that isolated myocytes treated with trimetazidine release less lactate dehydrogenase (LDH), even when exposed to oxidant agents (like oxygen peroxide $-\mathrm{H}_{2} \mathrm{O}_{2}$ ). In this experimental model, trimetazidine did not change ATP production, either during hypoxia or reoxygenation. This effect of trimetazidine was attributed by the authors not to its antioxidant properties, but to changes in lipid metabolism (Fantini et al., 1994). In other models of ischemia-reperfusion, it has been demonstrated that the impact of ischemia on the mitochondrial respiratory chain mainly occurs at the level of complex I (Veitch et al., 1992).

In ischemic models, a decrease in intracellular acidosis can be expected, providing that trimetazidine is present in the perfusion media (Renaud, 1988). This raises the hypothesis that trimetazidine acts on heart mitochondria, thus allowing a better use of the limited amount of $\mathrm{O}_{2}$ available during ischemia, by mechanisms not yet fully understood, but which may involve a preferential use of glucose and/or a decrease in reactive oxygen species production (Maupoil et al., 1990). In ischemic hearts perfused with free fatty acids and trimetazidine, ATP levels have been shown to be preserved by some authors (Fantini et al., 1994) and decreased by others (Demaison et al., 1995).

Most of the knowledge gathered so far about the intracellular mechanisms of trimetazidine was obtained from animal models perfused with lipids. Because ischemia is associated with a metabolic "shift" from fatty acid oxidation to glucose oxidation, and since this metabolic "shift" is further enhanced by treatment with trimetazidine (Kantor et al., 2000), we decided to determine the influence of trimetazidine on the mitochondrial function of ischemic rat hearts perfused with glucose (without the confounding effect of lipids). We demonstrate that trimetazidine improves, in a specific way, mitochondrial complex I enzyme activity, resulting in a more efficient consumption of $\mathrm{O}_{2}$ together with a better preservation of the mitochondrial transmembrane electrochemical gradient. This increase in complex I enzyme activity may further explain the cardioprotective effect of trimetazidine beyond the reported "metabolic shift".

\section{Materials and methods}

This investigation conformed with the Guide for the Care and Use of Laboratory Animals published by the US National Institutes of Health-NIH publication No. 85-23, revised 1996 (National Research Council, 1996) and it was approved by the Ethics Committee of the Centre for Neuroscience and Cell Biology of Coimbra, where the experimental work took place. All compounds were the purest quality available and were ordered from Sigma (St. Louis, MO) or Merck (Darmstadt, Germany), except for trimetazidine, which was obtained from Servier (Courbevoie, France).

\subsection{Experimental groups}

Thirty female Wistar rats were randomly distributed over three experimental groups: control $(n=10)$, ischemia $(n=10)$ and ischemia + trimetazidine $25 \mu \mathrm{M}(n=10)$.

\subsection{Assembly of the ex-vivo perfusion system}

Wistar rats, weighing around $300 \mathrm{~g}$, were killed by $\mathrm{CO}_{2}$ inhalation. Each heart was rapidly excised and mounted in a Langendorff perfusion apparatus (model UP-100 from Hugo Sachs Elektronik, Germany), and then perfused at a constant flow $(25 \mathrm{~mL} / \mathrm{min})$ with a Krebs modified solution $(\mathrm{NaCl}$ $118 \mathrm{mM}, \mathrm{KCl} 4.7 \mathrm{mM}, \mathrm{KH}_{2} \mathrm{PO}_{4} 1.2 \mathrm{mM}, \mathrm{NaHCO}_{3} 25 \mathrm{mM}$, $\mathrm{MgSO}_{4} 1.2 \mathrm{mM}, \mathrm{Na}_{2}$ EDTA $0.5 \mathrm{mM}$, Glucose $10 \mathrm{mM}$ and $\left.\mathrm{CaCl}_{2} 3 \mathrm{mM} ; \mathrm{pH} 7.4\right)$, gassed with carbogen $\left(95 \% \mathrm{O}_{2-}\right.$ $5 \% \mathrm{CO}_{2}$ ). Temperature was continuously monitored and maintained at $37{ }^{\circ} \mathrm{C}$ (via a thermostatic bath) throughout the perfusion period (180 min for the control group and $60 \mathrm{~min}$ for the remaining groups); $\mathrm{pH}$ was also continuously monitored with a $\mathrm{pH}$ electrode and kept stable at 7.357.45 during this period. Trimetazidine $(25 \mu \mathrm{M})$ was added to the Krebs modified solution in the respective experimental group. Left ventricular pressure and heart rate were recorded at regular intervals.

After the initial perfusion, all rat hearts, with the exception of the control group, were submitted to $120 \mathrm{~min}$ of ischemia at $37{ }^{\circ} \mathrm{C}$ in a solution identical to that of the perfusion period, but without glucose and carbogen (replaced by nitrogen).

\subsection{Preparation of the biological material}

\subsubsection{Isolation of rat heart mitochondria}

At the end of the perfusion and ischemia periods, the mitochondrial fraction from each rat heart was prepared according to the method described by Rickwood et al. (1987). All the parameters of mitochondrial function were assessed in the isolated mitochondrial fraction.

\subsection{Laboratory methods used}

The laboratory methods used in this experimental protocol have been described earlier (Monteiro et al., 2003).

\subsubsection{Statistical analysis}

Results are presented as means \pm standard error (raw data or expressed as percentage of control), for the number of 
results indicated. Results were analyzed using the Student $t$-test. The level of significance used was $P<0.05$.

\section{Results}

Oxidative phosphorylation is very sensitive to ischemia, which affects mitochondrial coupling, as demonstrated by the decrease in the respiratory rate when compared with mitochondria of the control group $(2.68 \pm 0.25$ versus $1.51 \pm 0.13 ; P=0.0002)$. Trimetazidine $(25 \mu \mathrm{M})$ significantly protected heart mitochondria from the deleterious effects of ischemia on respiratory control rate (Fig. 1), decreasing $\mathrm{O}_{2}$ consumption during state 4, that is, after ADP phosphorylation (from $16.73 \pm 2$ to $10.21 \pm 2.1 \mathrm{nmol} \mathrm{O}_{2} / \mathrm{min}$; $P=0.001$ ), when glutamate/malate was used as energy substrate. This decrease resulted in a higher respiratory control rate in this experimental group (2.71 \pm 0.42 -Fig. 2$)$, significantly higher than that in the ischemic group ( $P=0.0002)$ and similar to that of the control group ( $P=$ n.s.).

When glutamate/malate was used as energy substrate, the electrical potential $(\Delta \Psi)$ decreased in a significant manner during ischemia, compared to that in the control group $(209.13 \pm 1.37$ versus $186.66 \pm 3.36 \mathrm{mV} ; P=0.000001)$. The same occurred when succinate $(211.32 \pm 3.24$ versus $187.23 \pm 4.74 \mathrm{mV} ; P<0.05)$ and ascorbate/TMPD $\left(N, N, N^{\prime}, N^{\prime}\right.$-Tetramethyl- $P$-phenylenodiamine) (209.09 \pm 2.53 versus $189.24 \pm 3.98 \mathrm{mV} ; P<0.05)$ were used. In mitochondria from trimetazidine-treated hearts, $\Delta \Psi$ increased significantly to $199.49 \pm 1.7 \mathrm{mV}(P=0.004)$ (Fig. $3 \mathrm{~A})$. Similar results were obtained when succinate (197.41 \pm 1.84 versus $187.23 \pm 4.74 \mathrm{mV} ; P=0.04)$ and ascorbate/TMPD (199.77 \pm 1.21 versus $189.24 \pm 3.98 \mathrm{mV}$; $P=0.01$ ) were used as energy substrates (Fig. 3A).

Another parameter evaluated was the amount of time needed to phosphorylate a fixed amount of ADP (250 nmol) - the so-called lag phase. The lower this value is, the quicker the phosphorylation cycle is completed. The lag phase was increased in mitochondria from hearts of the ischemic group, when compared to those from the control group, not only with glutamate/malate $(59.08 \pm 6.82$ versus $127.2 \pm 19.03 \mathrm{~s} ; P=0.001$ ), but also with succinate $(66.46 \pm 6.43$ versus $275.73 \pm 45.99 \mathrm{~s} ; P=0.0001)$ and ascor-

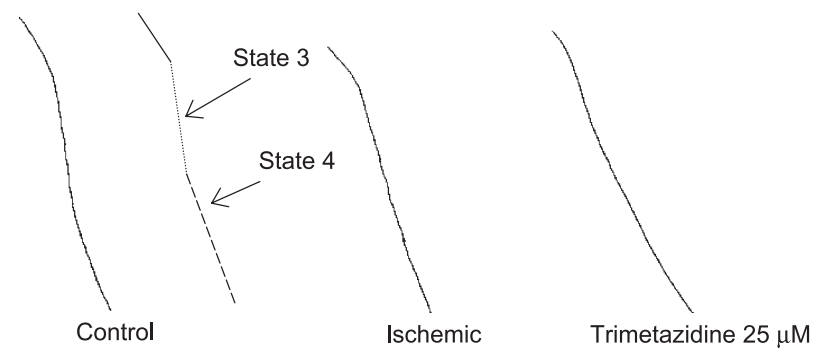

Fig. 1. Typical recording of $\mathrm{O}_{2}$ consumption during states 3 and 4 of mitochondrial respiration (using glutamate/malate as energy substrate).

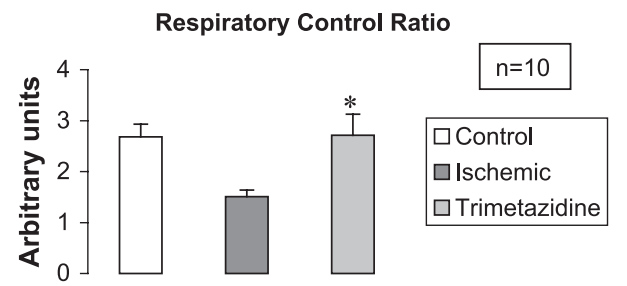

Fig. 2. Comparison of respiratory control ratio in rat heart mitochondria (control, ischemic and trimetazidine groups) with glutamate/malate as energy substrate. ${ }^{*} P<0.05$ versus ischemic group; values are expressed in arbitrary units.

bate/TMPD $(31.5 \pm 2.11$ versus $62.4 \pm 11.63 \mathrm{~s} ; P=0.001)$. Compared to the ischemic group (Fig. 3B), mitochondria from hearts treated with trimetazidine $25 \mu \mathrm{M}$ had a similar lag phase, independent of the energy substrate used: glutamate/ malate $(132.1 \pm 21.39$ versus $127.2 \pm 19.03 \mathrm{~s} ; P=\mathrm{n} . \mathrm{s}$.), succinate $(250.62 \pm 17.05$ versus $275.73 \pm 45.99 \mathrm{~s} ; P=$ n.s. $)$ or ascorbate/TMPD $(48.3 \pm 6.39$ versus $62.4 \pm 11.63 \mathrm{~s} ; P=$ n.s. $)$.

We also determined the energy charge ([ATP $]+0.5^{*}$ $[\mathrm{ADP}] /([\mathrm{ATP}]+[\mathrm{ADP}]+[\mathrm{AMP}]))$ obtained when using glutamate/malate, succinate and ascorbate/TMPD. As for other parameters, the mitochondria from the ischemic group generated a lower energy charge per phophorylation cycle, when compared to those of the control group, independent of the energy substrate used. The difference between the two groups reached statistical significance with glutamate/ malate $(0.965 \pm 0.005$ versus $0.94 \pm 0.01 ; P=0.04)$, but not with succinate $(0.79 \pm 0.051$ versus $0.64 \pm 0.04 ; P=$ n.s. $)$ and ascorbate/TMPD $(0.7 \pm 0.04$ versus $0.59 \pm 0.03 ; P=$ n.s. $)$. The energy charge of mitochondria from the trimetazidinetreated group, was similar ( $P=$ n.s.) to that of the ischemic hearts not treated with trimetazidine (Fig. 3C), regardless of the energy substrate used (glutamate/malate: 0.91 \pm 0.04 ; succinate: $0.65 \pm 0.09$; ascorbate/TMPD: $0.59 \pm 0.12$ ).

Finally, we evaluated the enzyme activity of complexes I, II-III and IV. A statistically significant difference $(P<0.05)$ between mitochondria from ischemic and control hearts, was observed for all four enzyme complexes evaluated (complex I: $100 \%$ versus $63.23 \pm 5.18 \%$; complexes II-III: $14370 \pm 1174$ versus $8592 \pm 663 \mathrm{nmol}$ succinate oxidized/ $\mathrm{min} / \mathrm{mg}$ protein; complex IV: $853 \pm 66$ versus $608 \pm 58 \mathrm{nmol}$ $\mathrm{O}_{2}$ consumed $/ \mathrm{min} / \mathrm{mg}$ protein; ATP synthase: $795 \pm 75$ versus $426 \pm 35 \mathrm{nmol} \mathrm{H}{ }^{+}$released $/ \mathrm{min} / \mathrm{mg}$ protein). Mitochondria from hearts submitted to ischemia in the presence of trimetazidine showed a significant increase in the complex I enzyme activity, when compared with both those from the ischemic group $(153.85 \pm 10.58 \%$ versus $63.23 \pm$ $5.18 \% ; P=0.0001)$ and the control group $(153.85 \pm 10.58 \%$ versus $100 \% ; P=0.02$ ). Trimetazidine induced a non-statistically significant increase in the enzyme activity of complexes II-III, when compared with mitochondria from ischemic hearts not treated with trimetazidine $(9357 \pm 593$ versus $8592 \pm 663 \mathrm{nmol}$ succinate oxidized $/ \mathrm{min} / \mathrm{mg}$ protein; $P=$ n.s.). Complex IV $\left(606 \pm 40\right.$ versus $608 \pm 58 \mathrm{nmol} \mathrm{O}_{2}$ consumed $/ \mathrm{min} / \mathrm{mg}$ protein) and ATP synthase (432 \pm 83 
A
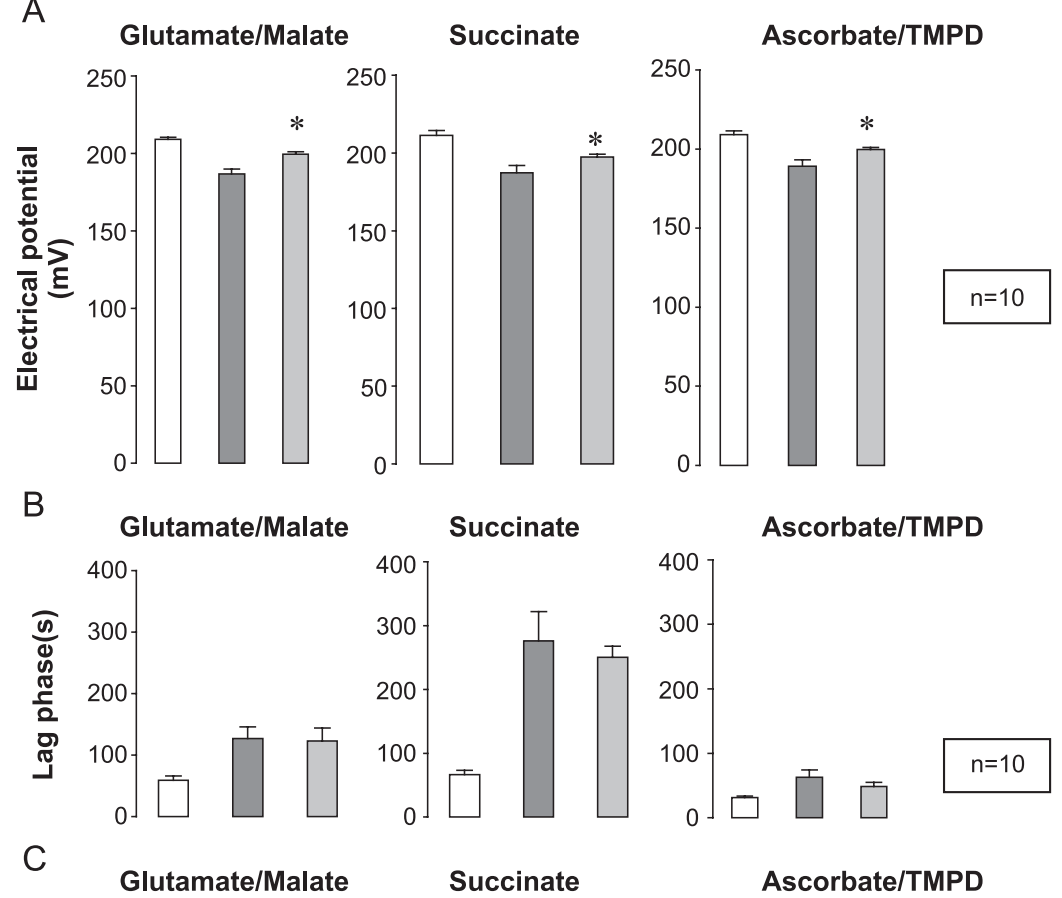

Succinate

Ascorbate/TMPD
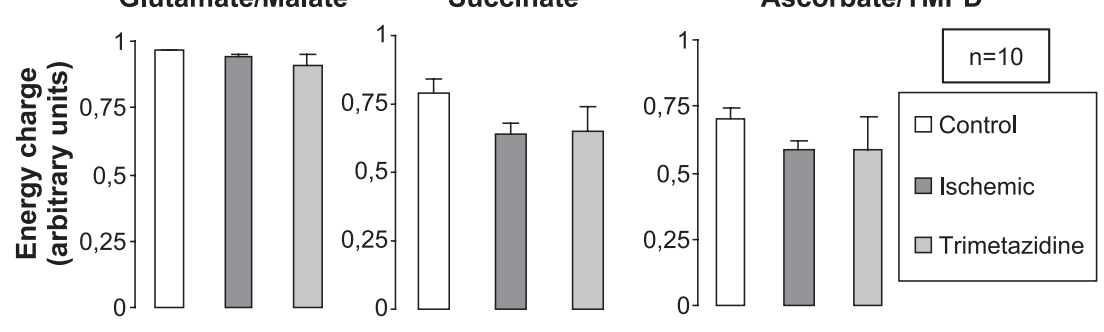

Fig. 3. (A) Comparison of maximal electrical potential $(\Delta \Psi)$ in the control, ischemic and trimetazidine-treated hearts, after the addition of the energy substrate to the reaction medium containing rat heart mitochondria. Values are expressed in $\mathrm{mV}$. ${ }^{*} P<0.05$ versus the ischemic group. (B) Comparison of the phosphorylation lag phase in the experimental groups (control, ischemic and trimetazidine) for each of the energy substrates studied, after the addition of 250 nmol of ADP to the medium containing rat heart mitochondria and the substrate. Values are expressed in seconds. ${ }^{*} P<0.05$ versus the ischemic group. (C) Comparison of the energy charge values (obtained by high pressure liquid chromatography) in the experimental groups (control, ischemic and trimetazidine) for each of the energy substrates studied, after the addition of $250 \mathrm{nmol}$ of ADP (62.5 when using ascorbate/TMPD) to the medium containing rat heart mitochondria and the energy substrate. Values are expressed in arbitrary units. ${ }^{*} P<0.05$ versus the ischemic group.

versus $426 \pm 35 \mathrm{nmol} \mathrm{H}^{+}$released/min/mg protein) enzyme activities were similar $(P=$ n.s. $)$ in mitochondria from the trimetazidine and ischemic groups (Fig. 4A, B, C and D).

\section{Discussion}

Since it has been shown that during ischemia there is an increased use of glucose as the myocardial energy source, when studying the impact of a drug on cardiac mitochondrial metabolism during ischemia it is important to perfuse the hearts with glucose. Furthermore, because we wanted to investigate the impact of trimetazidine on cardiomyocyte function beyond the already proven "metabolic shift", it was imperative not to use lipids as the energy substrate.

Trimetazidine $(25 \mu \mathrm{M})$ had a global positive effect, but this effect was of different magnitude when considering the phosphorylation and oxidation systems. Trimetazidine had little effect on the phosphorylation system (energy charge, lag phase and ATP synthase enzyme activity). These results are in accordance with those of other investigators, who showed that trimetazidine was unable to improve the mitochondrial energy charge in animal models of myocardial ischemia (Fantini et al., 1994; Hugtenburg et al., 1989). During ischemia, trimetazidine was able to significantly improve variables related to the status of the mitochondrial respiratory chain. There was a significant increase in the respiratory control rate and, mainly, an increase in complex I enzyme activity. In similar experimental models, but in which a perfusion medium without glucose (replaced by fatty acids) was used, trimetazidine had a negative impact on the respiratory control rate (Fantini et al., 1994). These different results could be explained by the preferential use, by ischemic hearts, of glucose as energy substrate (instead of fatty acids). This preferential use of glucose is increased in the presence of trimetazidine.

A more detailed analysis of components of respiratory control ratio $\left(\mathrm{O}_{2}\right.$ consumption in states 3 and 4 of 
A

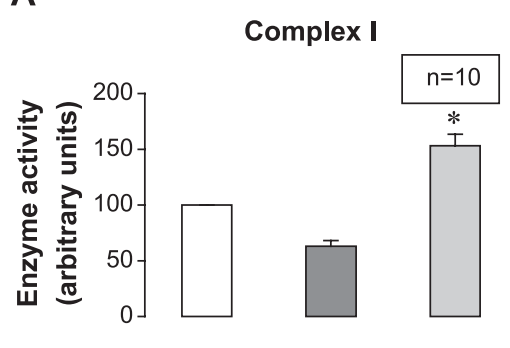

B

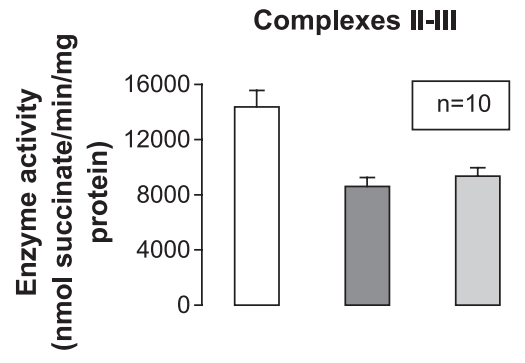

C

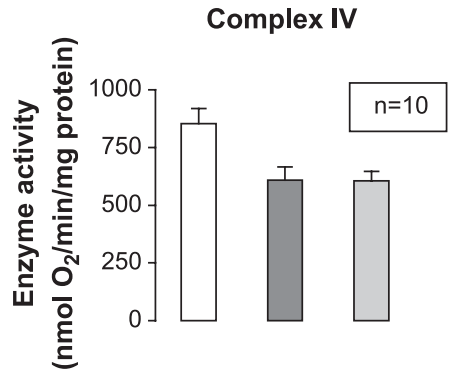

D

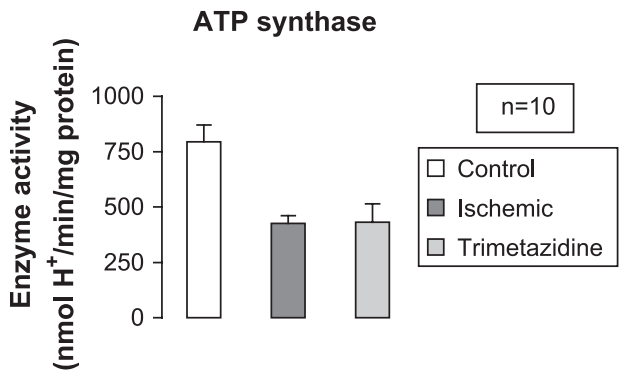

Fig. 4. Comparison of the enzyme activity of rat heart mitochondria of the experimental groups (control, ischemic and trimetazidine), for each of the complexes studied (I, II-III, IV and ATP synthase), in the presence of the substrate of each complex (NADH, succinate, ascorbate/TMPD or ATP-Mg ${ }^{2+}$, respectively).

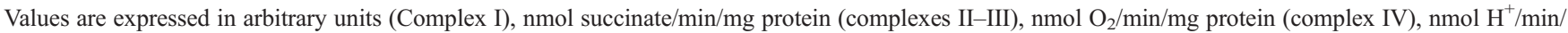
mg protein (ATP synthase). A-complex I; B — complexes II-III; C-complex IV; D-ATP synthase. ${ }^{*} P<0.05$ versus the ischemic group.

mitochondrial respiration) showed that the protective effect of trimetazidine under these experimental conditions seemed to be mainly due to a more efficient use of $\mathrm{O}_{2}$ during state 4 of mitochondrial respiration. Other authors have demonstrated, in similar animal models, different results using fatty acids as substrate. Fantini et al. (1994) observed a decrease in $\mathrm{O}_{2}$ consumption during state 3. The explanation for these differences could be related to the different substrates used and to the fact that ischemic hearts prefer to use glucose as substrate (situation improved by trimetazidine itself).

The effect of trimetazidine on mitochondrial respiration observed in our experimental model could be explained by an increase in the activity of complex I during ischemia (with a consequent decrease in the production of reactive oxygen species). This enzyme-based mechanism could explain the antioxidant properties of trimetazidine already described by Maupoil et al. (1990). The confirmation of our findings in future studies may make it possible to explain the molecular mechanisms involved in that antioxidant effect (until now mainly regarded as a mere consequence of the trimetazidine-induced "metabolic shift") (Kantor et al., 2000) and to fully explain the anti-ischemic effect of trimetazidine, as these new data clearly demonstrate that it goes far beyond the "metabolic shift" (being also present in an experimental model in which the hearts were not exposed to free fatty acids).

The decrease in reactive oxygen species production could also explain the higher membrane electrical potential found in the mitochondria from hearts treated with trimetazidine. It was previously demonstrated that reactive oxygen species are responsible for peroxidation, which compromises membrane integrity (Maridonneau-Parini and Harpey, 1985) and, consequently, the maintenance of normal electrochemical gradients. In fact, Fantini et al. (1994) have already suggested that trimetazidine preserves membrane integrity. This effect could be determined by the direct antioxidant effect, by a membrane-stabilizing effect unrelated to antioxidant mechanisms or by a decrease in cellular activity (in metabolic and/or mechanical terms), supposedly related to the negative chronotropic and inotropic effects of trimetazidine (Lavanchy et al., 1987).

\section{Conclusions}

By promoting the activity of the mitochondrial respiratory chain complex I in ischemic cardiomyocytes, trimetazidine enables a more efficient $\mathrm{O}_{2}$ consumption during the metabolism of glucose. This action could lead to a decrease in reactive oxygen species production and to an increase in mitochondrial membrane integrity, resulting in a better preservation of the transmembrane electrochemical gradient. All these effects have a positive impact on mitochondrial homeostasis and, therefore, on the metabolism of cardiomyocytes during ischemia.

It is clearly demonstrated that, during acute ischemia, the positive effects of trimetazidine are not primarily due to an increase in ATP production, but to an impact on the mitochondrial respiratory chain, increasing complex I activity and thus decreasing futile $\mathrm{O}_{2}$ consumption and reactive oxygen species production. 


\section{Acknowledgement}

This study was supported by grants from the Portuguese Society of Cardiology and the Procardio.

\section{References}

Dalla-Volta, S., Maraglino, G., Della-Valentina, P., Desideri, A., 1990. Comparison of trimetazidine with nifedipine in effort angina: a doubleblind cross-over study. Cardiovasc. Drugs Ther. 4 (suppl 4), 853-859.

Demaison, L., Fantini, E., Sentex, E., Grynberg, A., Athias, P., 1995. Trimetazidine: in vitro influence on heart mitochondrial function. Am. J. Cardiol. 76, B31-B37.

Detry, J.M., Sellier, P., Pennaforte, S., Cokkinos, D., Dargie, H., Mathes, P., 1994. Trimetazidine: a new concept in the treatment of angina. Comparison with propranolol in patients with stable angina. Trimetazidine European Multicenter Study Group. Br. J. Clin. Pharmacol. 37, $279-288$.

Fabiani, J.N., Ponzio, O., Massonet-Castel, S., Paris, M., Chevalier, P., Jebara, V., Carpentier, A., 1992. Cardioprotective effect of trimetazidine during coronary artery graft surgery. J. Cardiovasc. Surg. 33, 486-491.

Fantini, E., Demaison, L., Sentex, E., Grynberg, A., Athias, P., 1994. Some biochemical aspects of the protective effect of trimetazidine on rat cardiomyocytes during hypoxia and reoxygenation. J. Mol. Cell. Cardiol. 26, 949-958.

Hugtenburg, J.G., Jap, T.J.W., Mathy, M.J., Van Heiningen, P.N.M., Bohnen, V.A., Heijnis, J.B., Boddedke, H.W.G.M., Van Zwieten, P.A., 1989. Cardioprotective effect of trimetazidine and nifedipine in guinea pig hearts subjected to ischemia. Arch. Int. Pharmacodyn. Ther. 300, 186-208.

Kantor, P.F., Lucien, A., Kozak, R., Lopaschuk, G.D., 2000. The antianginal drug trimetazidine shifts cardiac energy metabolism from fatty acid oxidation to glucose oxidation by inhibiting mitochondrial long-chain 3-ketoacyl coenzyme A thiolase. Circ. Res. 86, $487-489$
Kober, G., Buck, T., Sievert, H., Vallbracht, C., 1992. Myocardial protection during percutaneous transluminal coronary angioplasty: effects of trimetazidine. Eur. Heart J. 13, 1109-1115.

Lavanchy, N., Martin, J., Rossi, A., 1987. Anti-ischemic effects of trimetazidine: ${ }^{31} \mathrm{P}$ NMR spectroscopy in the isolated rat heart. Arch. Int. Pharmacodyn. 289, 97-110.

Maridonneau-Parini, I., Harpey, C., 1985. Effects of trimetazidine on membrane damage induced by oxygen free radicals in human red cells. Brit. J. Clin. Pharmacol. 20, 148-151.

Maupoil, V., Rochette, L., Tabard, A., Clauser, P., Harpey, C., 1990. Evolution of free radical formation during low-flow ischemia and reperfusion in isolated rat heart. Cardiovasc. Drugs. Ther. 4, 791-795.

Monteiro, P., Duarte, A.I., Moreno, A., Gonçalves, L.M., Providência, L.A., 2003. Carvedilol improves energy production during acute global myocardial ischaemia. Eur. J. Pharmacol. 482, 245-253.

Renaud, J.F., 1988. Internal $\mathrm{pH}, \mathrm{Na}^{+}$and $\mathrm{Ca}^{2+}$ regulation by trimetazidine during cardiac cell acidosis. Cardiovasc. Drugs Ther. 1, 677-686.

Rickwood, D., Wilson, M.T., Darley-Usmar, V.M., 1987. Isolation and characteristics of intact mitochondria-isolation of mitochondria from mammalian cells. In: Darley-Usmar, V.M., Rickwood, D., Wilson, M.T. (Eds.), Mitochondria: a practical approach. IRL Press, Oxford, England, pp. 4-5.

Sellier, P., Audouin, P., Payen, B., Corona, P., Duong, T.C., Ourbak, P., 1987. Acute effects of trimetazidine evaluated by exercise testing. Eur. J. Clin. Pharmacol. 33, 205-207.

Sentex, E., Sergiel, J.P., Lucien, A., Grynberg, A., 1998. Is the cytoprotective effect of trimetazidine associated with lipid metabolism? Am. J. Cardiol. 82, 18K-24K.

Stanley, W.C., Lopaschuk, G.D., Hall, J.L., McCormak, J.G., 1997. Regulation of myocardial carbohydrate metabolism under normal and ischaemic conditions. Cardiovasc. Res. 33, 243-257.

Veitch, K., Hombroeckx, A., Caucheteux, D., Pouleur, H., Hue, L., 1992. Global ischemia induces a biphasic response of the mitochondrial respiratory chain. Anoxic pre-perfusion protects against ischaemic damage. Biochem. J. 281, 709-715.

Veitch, K., Maisin, L., Hue, L., 1995. Trimetazidine effects on the damage to mitochondrial functions caused by ischemia and reperfusion. Am. J. Cardiol. 76, 25B-30B. 